\title{
Afferent-Specific AMPA Receptor Subunit Composition and Regulation of Synaptic Plasticity in Midbrain Dopamine Neurons by Abused Drugs
}

\author{
Cameron H. Good and Carl R. Lupica \\ Electrophysiology Research Section, Cellular Neurobiology Branch, National Institute on Drug Abuse Intramural Research Program, National Institutes of \\ Health, Baltimore, Maryland 21224
}

\begin{abstract}
Ventral tegmental area (VTA) dopamine (DA) neurons play a pivotal role in processing reward-related information and are involved in drug addiction and mental illness in humans. Information is conveyed to the VTA in large part by glutamatergic afferents that arise in various brain nuclei, including the pedunculopontine nucleus (PPN). Using a unique rat brain slice preparation, we found that PPN stimulation activates afferents targeting GluR2-containing AMPA receptors (AMPAR) on VTA DA neurons, and these afferents did not exhibit long-term depression (LTD). In contrast, activation of glutamate afferents onto the same DA neurons via stimulation within the VTA evoked EPSCs mediated by GluR2-lacking AMPARs that demonstrated LTD or EPSCs mediated by GluR2-containing AMPA receptors that did not express LTD. Twenty-four hours after single cocaine injections to rats, GluR2-lacking AMPARs were increased at both PPN and local VTA projections, and this permitted LTD expression in both pathways. Single injections with the main psychoactive ingredient of marijuana, $\Delta^{9}$-tetrahydrocannabinol $\left(\Delta^{9}\right.$-THC), increased GluR2-lacking AMPA receptors and permitted LTD in only the PPN pathway, and these effects were prevented by in vivo pretreatment with the cannabinoid CB1 receptor antagonist AM251. These results demonstrate that cocaine more globally increases GluR2-lacking AMPA receptors at all glutamate synapses on VTA dopamine neurons, whereas $\Delta^{9}$-THC selectively increased GluR2-lacking AMPA receptors at subcortical PPN synapses. This suggests that different abused drugs may exert influence over distinct sets of glutamatergic afferents to VTA DA neurons which may be associated with different reinforcing or addictive properties of these drugs.
\end{abstract}

\section{Introduction}

VTA DA neurons play a pivotal role in processing rewardrelevant information and are involved in addiction and mental illness in humans (Wise, 2005). Environmental stimuli can cause DA neurons to shift firing patterns from pacemaker to highfrequency bursts of activity, leading to large increases in DA release in target areas (Gonon, 1988; Schultz, 1998; Hyland et al., 2002). Glutamate activation of ionotropic AMPARs on DA neurons appears to play an important role in this shift to bursting (Overton and Clark, 1997; Kitai et al., 1999; Georges and AstonJones, 2002; Floresco et al., 2003). Acute and long-term exposure to abused drugs can alter VTA glutamatergic afferents and produce enduring changes in DA neuron activity (Wolf et al., 2004). Thus, these VTA afferents exhibit changes, such as long-term potentiation (LTP) or LTD after exposure to abused drugs in vivo, and following local electrical stimulation in vitro (Bonci and Malenka, 1999; Jones et al., 2000; Mansvelder and McGehee,

Received March 24, 2010; accepted April 22, 2010.

This work was supported by The U.S. Department of Health and Human Services, the National Institutes of Health, and the National Institute on Drug Abuse Intramural Research Program. We thank Drs. Cristina Bäckman and YaJun Zhang for performing DAT immunohistochemistry and biocytin reconstructions of VTA neurons, and Drs. Alex Hoffman, Eisuke Koya, and Bruce Hope for helpful comments on the manuscript.

Correspondence should be addressed to Carl R. Lupica, NIH/NIDA-IRP, 333 Cassell Drive, Baltimore, MD, 21224. E-mail: clupica@intra.nida.nih.gov.

DOI:10.1523/JNEUROSCI.1507-10.2010

Copyright $\odot 2010$ the authors $\quad 0270-6474 / 10 / 307900-10 \$ 15.00 / 0$
2000; Ungless et al., 2001; Saal et al., 2003; Bellone and Lüscher, 2005; Kauer and Malenka, 2007).

Although synaptic plasticity represents an intriguing substrate to explain lasting effects of abused drugs on reward circuits, there remain unresolved issues relating to this phenomenon. The VTA receives glutamatergic inputs from diverse brain nuclei (Geisler et al., 2007), and the origin of the afferents undergoing plasticity in vitro remain largely unknown. This is because most nuclei sending afferents to the VTA are not present within conventional brain slice preparations, and because many afferents are severed during the slicing procedure. Although cortical afferents provide input to the VTA, major glutamatergic afferents originating in subcortical nuclei also provide substantial direct input to VTA DA neurons (Omelchenko and Sesack, 2007). The PPN is one subcortical nucleus providing ascending glutamatergic, GABAergic, and cholinergic input to VTA DA neurons (Cornwall et al., 1990; Oakman et al., 1995; Geisler and Zahm, 2005; Wang and Morales, 2009). Consistent with its projections to the VTA, PPN stimulation increases DA neuron activity and DA concentrations in projection areas (Klitenick and Kalivas, 1994; Floresco et al., 2003), suggesting that it influences reward and motivational functions of the VTA. This is supported by studies showing that PPN lesions disrupt learned associations between primary rewards and neutral stimuli (Inglis et al., 2000), conditioned-place preference to opiates and psychostimulants (Bechara and van der Kooy, 1989; Olmstead and 

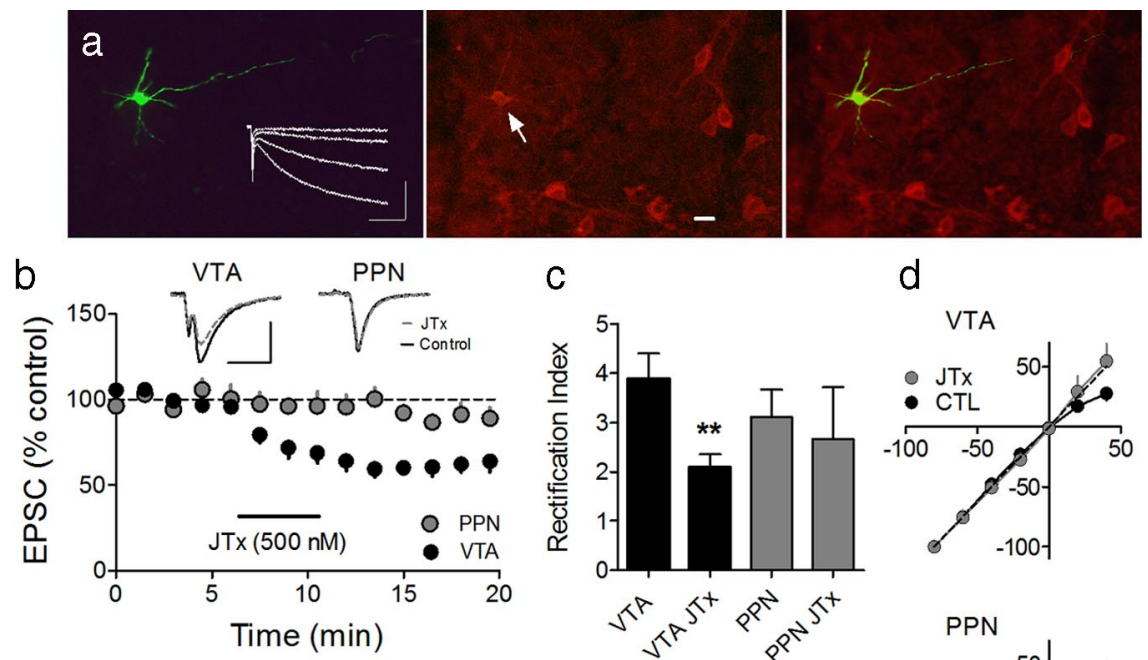

C
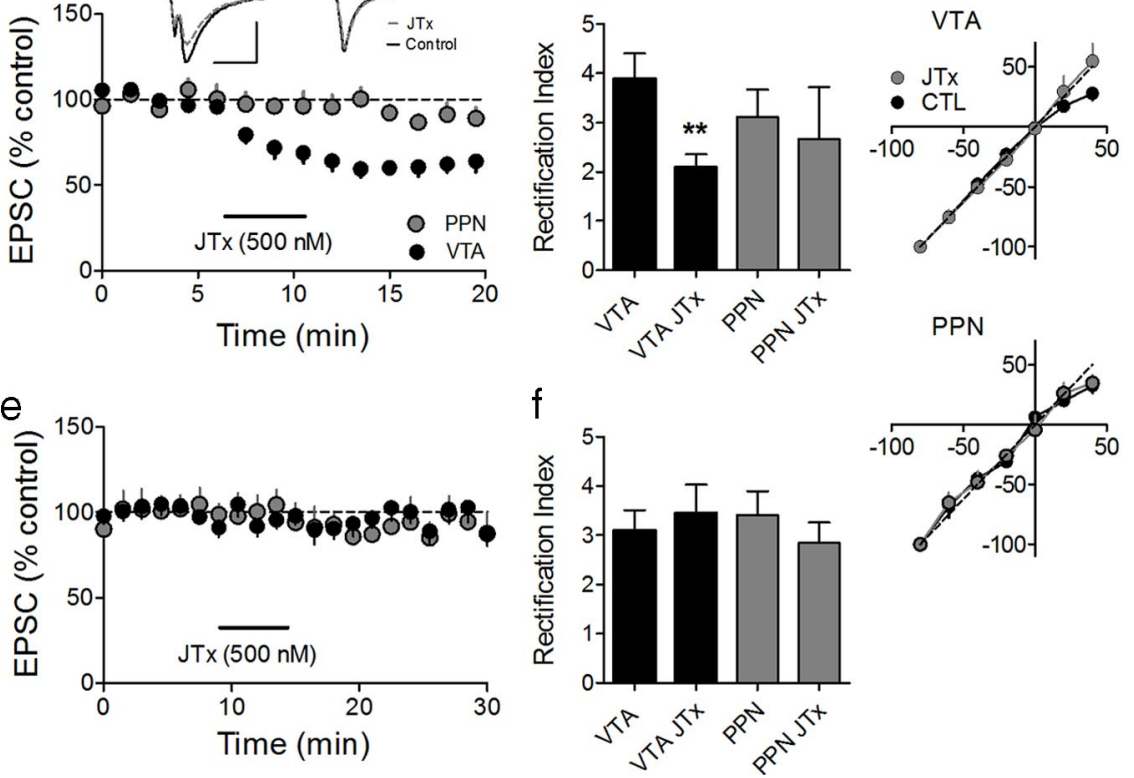

Figure 1. JTx inhibition of EPSCs evoked via intra-VTA, but not PPN stimulation, indicates the absence or presence of the GluR2 AMPAR subunit, respectively, at these synapses on VTA DA neurons. $\boldsymbol{a}$, Photomicrograph of a biocytin-filled (left) VTA neuron that expressed the DAT (center, arrow), and a superimposition of these images (right). Also shown are membrane currents elicited by hyperpolarizing voltage steps, indicating robust $I_{h}$ (left, inset; scale bars, $200 \mathrm{~ms}$ and $200 \mathrm{pA}$ ). Scale bar, $20 \mu \mathrm{m}$. $\boldsymbol{b}$, Mean time course of JTx (horizontal bar) effects on EPSCs evoked via intra-VTA or PPN stimulation in the same VTA DA neurons $(n=14)$. Inset, Representative EPSCs during control and JTx application ( $n=3-5$ EPSCs per average; scale bars, $10 \mathrm{~ms}, 100 \mathrm{pA}$ ). Data shown in $\boldsymbol{b}$ represent only neurons in which intra-VTA-evoked EPSCs were inhibited by JTx (16/25, 64\%). e, Absence of JTx effect on intra-VTA or PPN-evoked EPSCs in a subpopulation of VTA DA neurons (9/25,36\%). c, RI of EPSCs evoked via intra-VTA or PPN stimulation, before and during JTx application. There was a significant decrease in RI of the intra-VTA (paired $t$ test, $p<0.01$ ), but not the PPN-evoked, EPSCs in a subset of cells, whereas there was no change in the RI measured at either pathway in the remaining neurons $(\boldsymbol{f}$, ANOVA, ns). $\boldsymbol{d}, I-V$ curves measured before and after JTx application from the same cells as in $\boldsymbol{b}$ and $\boldsymbol{c}$.

Franklin, 1993), amphetamine-induced sensitization (Nelson et al., 2007), and self-administration of heroin (Olmstead et al., 1998).

Despite its potential importance in mediating the effects of abused drugs, the PPN inputs to the VTA are poorly characterized. To address this, we developed a brain slice preparation that preserves PPN neurons and projections to VTA DA cells, and demonstrated that PPN-activated glutamate synapses could be studied independently of those activated by intra-VTA stimulation (Good and Lupica, 2009). We used this preparation in the present study to examine distinct glutamatergic inputs to VTA DA neurons, determined their susceptibility to LTD, and whether these properties are altered by cocaine and the psychoactive constituent of marijuana, $\Delta^{9}$-tetrahydrocannabinol ( $\Delta^{9}$-THC).

\section{Materials and Methods}

All animal procedures were conducted under National Institutes of Health (NIH) guidelines using the NIH Guide for the Care and Use of Laboratory Animals, which is based upon the United States Animal Welfare Act. The protocols were approved by the Institutional Animal Care and Use Committee (National Institute on Drug Abuse, Intramural Research Program, Baltimore, MD), which is accredited by the International Association for Assessment and Accreditation of Laboratory Animal Care. d

Brain slice preparation. Parasagittal brain slices containing the PPN and the VTA were prepared as previously described (Good and Lupica, 2009). Briefly, juvenile male (14 to $19 \mathrm{~d}$ old) rats were removed from a shared home cage no more than 15 min before decapitation. Animals were decapitated using a guillotine and the brain subsequently removed from the cranium and immediately transferred to a beaker containing oxygenated $\left(95 \% \mathrm{O}_{2} / 5 \% \mathrm{CO}_{2}\right)$, ice-cold $\left(2-4^{\circ} \mathrm{C}\right)$, artificial CSF (aCSF) of the following composition ( $\mathrm{mM})$ : sucrose, 194; $\mathrm{NaCl}, 30 ; \mathrm{KCl}, 4.5 ; \mathrm{MgCl}_{2}, 1 ; \mathrm{NaH}_{2} \mathrm{PO}_{4}$ 1.2; glucose, 10; $\mathrm{NaHCO}_{3}, 26$; ascorbic acid, 0.4. The brains were then blocked for slicing as described previously (Good and Lupica, 2009). The remaining block of tissue was glued onto the cutting stage of a vibrating tissue slicer (Leica, VT1000) and submersed in ice-cold, oxygenated aCSF. The brain was positioned with the right hemisphere down and the thalamus facing the cutting blade. Two slices (280 $\mu \mathrm{m})$ containing both the VTA and PPN were obtained from each rat using this procedure. The slices were transferred to an oxygenated holding chamber $\left(31^{\circ} \mathrm{C}\right)$ and allowed to recover for at least $1 \mathrm{~h}$ before recording.

Electrophysiology. One brain slice was transferred to a heated recording chamber $\left(31-33^{\circ} \mathrm{C}\right)$ and superfused with aCSF $(2 \mathrm{ml} /$ min) containing the following (in $\mathrm{mM}$ ): $\mathrm{NaCl}$, 126; KCl, 3; $\mathrm{MgCl}_{2}, 1.5 ; \mathrm{CaCl}_{2}, 2.4 ; \mathrm{NaH}_{2} \mathrm{PO}_{4}$, 1.2; glucose, $11 ; \mathrm{NaHCO}_{3}, 26$. Visualization of VTA neurons was performed with an upright microscope (Zeiss Axioscope), modified to provide a gradient-contrast image using infrared illumination. Recording electrodes (3-5 $\mathrm{M} \Omega$ ) contained the following (in $\mathrm{mm}$ ): $\mathrm{CsCl}$, 130; $\mathrm{NaCl}, 4 ; \mathrm{MgCl} 2,2$; EGTA, 1.1; HEPES, 5; $\mathrm{Na}_{2}$-ATP, $2 ; \mathrm{Na}_{2}$-creatine phosphate, $5 ; \mathrm{Na}_{3}-$ GTP, 0.6; spermine, 0.1; biocytin, $0.125 \%$; $\mathrm{pH}=7.2$ using $\mathrm{KOH}$ (osmolarity $=270-274$ mOsm). Whole-cell voltage-clamp recordings were performed using an Axopatch 200B (Molecular Devices). Voltage steps and stimulation protocols were delivered using the Strathclyde electrophysiology software package (WCP, courtesy of Dr. John Dempster, Strathclyde University, Glasgow, UK; http://spider. science.strath.ac.uk/sipbs/software ses.htm) and an A/D board (ITC-18, Instrutech Corp.) residing in a personal computer.

Neurons were first identified visually, and then selected on the basis of a large $(>200 \mathrm{pA})$ hyperpolarization-activated inward current $\left(I_{\mathrm{h}}\right)$, as originally described by Johnson and North (1992). Although more recent evidence demonstrates that the presence of $I_{\mathrm{h}}$ is not an unequivocal marker of VTA DA neurons (Margolis et al., 2006), additional studies show that $\sim 90 \%$ of tyrosine hydroxylase $(\mathrm{TH})$-positive DA neurons in the VTA demonstrate large $I_{\mathrm{h}}$, and only $13 \%$ of the $I_{\mathrm{h}}$-positive cells do not express TH (Cameron et al., 1997). Therefore, the absolute number of non-DAergic cells demonstrating large $I_{\mathrm{h}}$ in the VTA is likely low (Grace and Onn, 1989; Johnson and North, 1992). To further address this, dopamine transporter (DAT) immunohistochemistry was performed in most biocytin-filled VTA neurons to confirm the colocalization of large $I_{\mathrm{h}}$ magnitude with DAT immunohistochemistry (Fig. $1 a$ ). To measure $I_{\mathrm{h}}$, neurons were voltage-clamped at $-60 \mathrm{mV}$ and voltage steps from -70 to $-110 \mathrm{mV}$ were used.

As previously described, synaptic currents were evoked using two bipolar tungsten electrodes (Frederick Haer) with a tip separation of 300 $\mu \mathrm{m}$; one placed on the posterior PPN and the other anterior to the VTA (Good and Lupica, 2009). After placing each of the stimulators, single 
pulses (0.2 ms duration) were alternated between the intra-VTA and PPN stimulators, allowing $45 \mathrm{~s}$ between each alternating stimulus, and $90 \mathrm{~s}$ between stimuli applied to the same pathway. In our previous study it was found that at interpulse intervals shorter than $90 \mathrm{~s}$ there was significant rundown of the PPN-evoked synaptic current (Good and Lupica, 2009).

To calculate the rectification index (RI), full $I / V$ curves relating EPSC amplitude to membrane voltage were generated at holding potentials between $-80 \mathrm{mV}$ and $+40 \mathrm{mV}$, in $20 \mathrm{mV}$ increments, with 3 responses arising from each pathway averaged at each holding potential. The peak mean EPSC amplitude obtained at $-80 \mathrm{mV}$ was then divided by the mean EPSC amplitude obtained at $+40 \mathrm{mV}$. In figures in which the polyamines JTx (Joro spider toxin) or NASPM (1-naphthylacetylspermine trihydrochloride) were used, or when LTD was measured, each point represents the mean \pm SEM of EPSCs recorded at each time point.

All LTD experiments were performed in the presence of picrotoxin, D-(-)-2-amino-5-phosphonopentanoic acid (D-AP5) and atropine to block $\mathrm{GABA}_{\mathrm{A}}$, NMDA and muscarinic acetylcholine receptors, respectively. The synaptic currents remaining in AP-5 and picrotoxin were fully blocked by 6,7-dinitroquinoxaline-2,3-dione (DNQX), indicating the involvement of AMPA receptors in these responses (data not shown).

Following $10 \mathrm{~min}$ of baseline recording, LTD was initiated by 5 trains of 5 pulses at $66 \mathrm{~Hz}$, delivered at $10 \mathrm{~s}$ intervals through one of the stimulating electrodes. If LTD was not observed after the first train of 66 $\mathrm{Hz}$ stimuli, at least one more set of trains was initiated to ensure that mGluRs were sufficiently activated. LTD was then assessed by returning to the alternating stimulation of the PPN and intra-VTA pathways at $45 \mathrm{~s}$ intervals.

Drug injections. Sprague Dawley rats (Charles River Laboratories) were injected intraperitoneally with $15 \mathrm{mg} / \mathrm{kg}$ cocaine, $10 \mathrm{mg} / \mathrm{kg} \Delta^{9}$-THC, 2 $\mathrm{mg} / \mathrm{kg}$ [N-(piperidin-1-yl)-5-(4-iodophonyl)-1-(2,4-dichlorophenyl)4-methyl-1H-pyrazole-3-carboxamide] (AM251), or $0.9 \%$ saline with a $28 \mathrm{G}$ insulin syringe. Following these injections, each animal was immediately returned to its home cage. Injection volumes were small (100-200 $\mu \mathrm{l})$ to minimize stress. AM251 was injected either $30 \mathrm{~min}$ before $\Delta^{9}$ THC or alone in another group of rats.

Immunohistochemistry. The colocalization of biocytin and the DAT was performed in brain slices containing a single biocytin filled VTA neuron from which a recording was made. These slices were fixed in $4 \%$ paraformaldehyde overnight and then transferred to PBS containing $18 \%$ sucrose solution. The slices were then resectioned on a cryostat $(20$ $\mu \mathrm{m})$, collected on slides, and washed in PBS $(2 \times 5 \mathrm{~min})$. Sections were then incubated in a blocking buffer comprised of PBS containing $4 \%$ BSA and $0.3 \%$ Triton $\mathrm{X}-100$ for $1 \mathrm{~h}$ at room temperature, then transferred for $24 \mathrm{~h}$ to a solution (at $4^{\circ} \mathrm{C}$ ) containing the DAT antibody (Millipore), diluted 1:100 in blocking buffer. Sections were then washed twice in PBS, and incubated in Alexa Fluor 568-conjugated goat antibody to rat (Invitrogen) for $2 \mathrm{~h}$ at room temperature. To detect biocytin, sections were washed twice in PBS and incubated for $1 \mathrm{~h}$ at room temperature in a streptavidin, Alexa Fluor 488 conjugate (Invitrogen), diluted 1:800 in blocking buffer.

Data analysis and statistics. Data in text and figures are represented by mean \pm SEM. Statistical analysis was performed using either a two-tailed Student's $t$ test or a one-way ANOVA for multiple comparisons using GraphPad Prism v5.01. $Z$ tests were used to compare proportions of cells that underwent LTD in control and drug treatment conditions. To determine whether EPSCs from individual neurons were significantly affected by a particular manipulation (e.g., LTD-inducing stimuli) we calculated the SEM of all EPSCs elicited at each pathway in all DA neurons during the control period, before the initiation of any manipulation (equivalent to $10 \mathrm{~min}$ of baseline data for each neuron). This value was doubled $(2 \times$ SEM) and compared with the change in EPSC amplitude measured between 30 and 40 min following a particular manipulation. Any cell exhibiting a change in EPSC amplitude exceeding $2 \times$ the control SEM for a given pathway (equivalent to a $10.2 \%$ change for the intra-VTA pathway, and a $15 \%$ change for the PPN pathway) was then categorized as a "responder," whereas EPSCs exhibiting no change, or a change in amplitude $<2 \times$ control SEM was categorized as a "nonresponder." This provided a statistical measure to test the null hy- pothesis that there was no change in EPSC amplitude following a manipulation. This statistical test is equivalent to establishing a $95 \%$ confidence interval based on the mean variance of all cells during the baseline period because $95 \%$ of the data should fall within 2 SEM, if there is no change in EPSC amplitude. Thus, assuming a normal distribution, any change in EPSC amplitude exceeding $2 \times$ SEM is thought to be significantly different from the mean because the probability of this occurring by chance is $\leq 5 \%$.

Drugs. Atropine, picrotoxin, JTx, spermine, biocytin and NASPM were purchased from Sigma-Aldrich. DNQX and 3,5 dihydroxyphenylglycine (DHPG) were supplied from Tocris Bioscience. D-AP5 was purchased from Ascent Scientific. Cocaine and $\Delta^{9}$-THC were obtained from the National Institute on Drug Abuse drug supply system. JTx, NASPM, DNQX and DHPG were applied through bath superfusion using calibrated syringe pumps (Razel Scientific Instruments).

\section{Results}

\section{GluR subunit differences in distinct VTA DA neuron afferent synapses}

Most AMPARs in the mature mammalian CNS are heteromeric tetramers, containing the subunits GluR1-GluR4 in varying combinations. AMPAR permeability to $\mathrm{Ca}^{2+}$ is governed by the presence or absence of the GluR2 subunit, such that AMPARs lacking GluR2 gate $\mathrm{Ca}^{2+}$, whereas those containing GluR2 are impermeable to $\mathrm{Ca}^{2+}$ (Isaac et al., 2007). The presence of GluR2 subunits in synaptic AMPARs can be inferred from the distinct biophysical properties of synaptic EPSCs and their sensitivity to polyamine channel blockers such as JTx, and its synthetic analog NASPM. Thus, EPSCs mediated by GluR2-containing AMPARs do not inwardly rectify and are insensitive to polyamines, whereas AMPARs lacking GluR2 (i.e., GluR1 homomers or GluR3/GluR1 heteromers) show inward rectification and are blocked by polyamines. The incorporation of GluR2 into AMPARs is regulated by synaptic activity, and the increase in $\mathrm{Ca}^{2+}$ permeability following its removal is involved in both transient and sustained changes in synaptic strength (Bellone and Lüscher, 2005, 2006; Isaac et al., 2007; Mameli et al., 2007).

We previously demonstrated in parasagittal brain slices containing the PPN and VTA that brief electrical stimulation of these nuclei can elicit AMPAR-mediated EPSCs possessing distinct physiological and pharmacological characteristics in the same VTA DA neurons (Good and Lupica, 2009). However, the subunit composition of the AMPARs at these independent synapses were not determined. Therefore, we first examined the effect of the selective blockers of GluR2-lacking AMPARs, JTx (500 nM) or NASPM $(25 \mu \mathrm{M})$, on EPSCs evoked via stimulation of the PPN or within the anterior VTA (intra-VTA) in the same VTA DA neurons (Fig. 1b,e). Whereas a subpopulation of intra-VTA-evoked EPSCs were inhibited by JTx $(n=16 / 25,64 \%)$, all EPSCs elicited through PPN stimulation were unaffected by this polyamine $(n=$ $19,0 \%)$. Thus, in the same VTA DA neurons, many glutamate synapses activated by intra-VTA stimulation lacked GluR2, whereas most or all PPN-activated glutamate afferents targeted AMPARs containing GluR2.

As a result of voltage-dependent blockade of GluR2-lacking AMPARs by endogenous polyamines, EPSCs mediated by these ion channels also demonstrate inward rectification at positive membrane potentials (Isaac et al., 2007). Thus, EPSCs recorded at $+40 \mathrm{mV}$ are smaller than those at $-80 \mathrm{mV}$, and the ratio of these EPSCs, known as the rectification index ( $\mathrm{RI}=$ mean peak EPSC $_{-80 \mathrm{mV}} /$ mean peak EPSC $_{+40 \mathrm{mV}}$ ), can be used as a biophysical marker for the presence or absence of GluR2-containing AMPARs. Therefore, to confirm the results obtained with the polyamine blockers, we measured the RI of EPSCs activated 

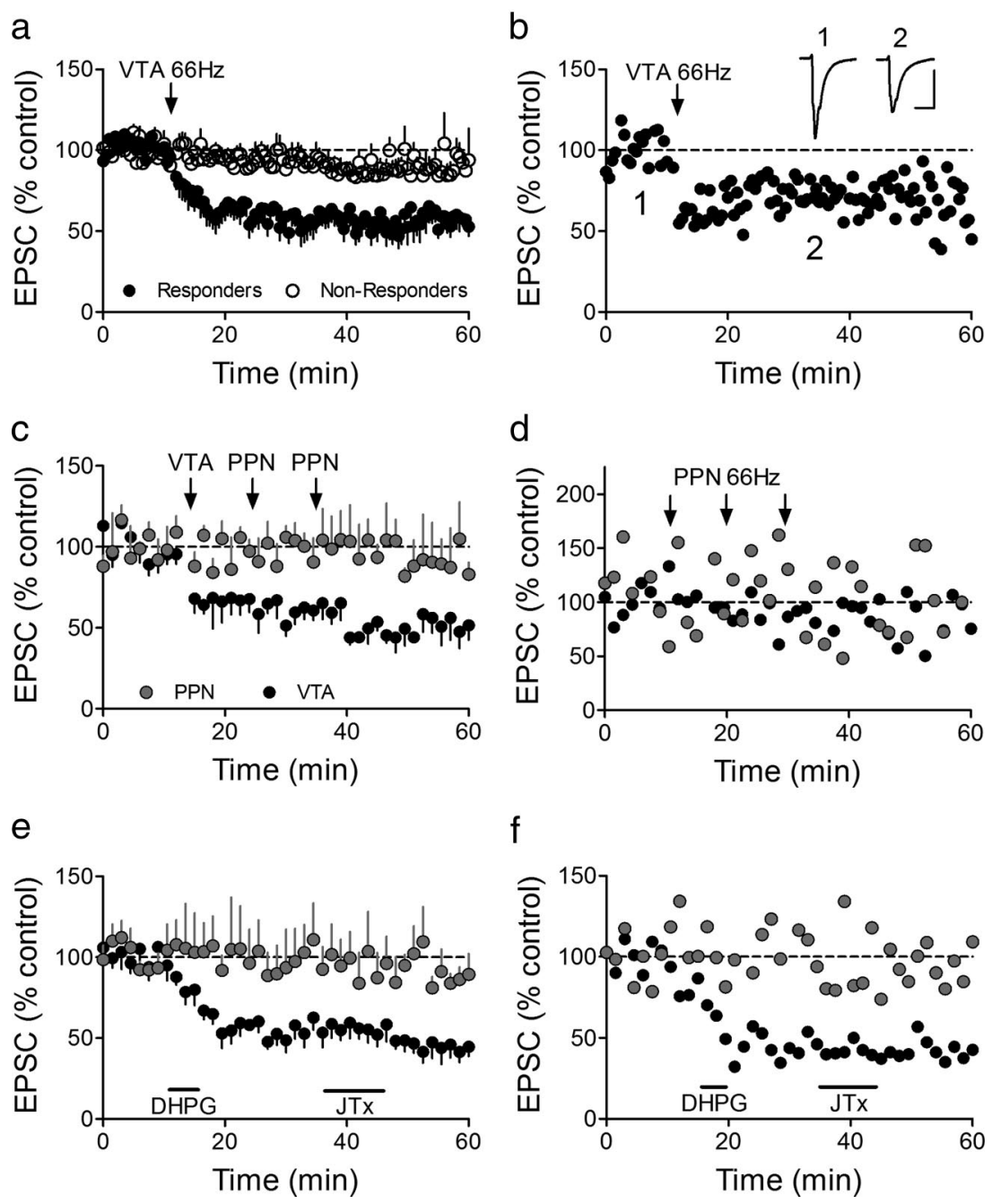

Figure 2. Selective LTD expression at intra-VTA-activated glutamate synapses. $\boldsymbol{a}$, Mean time course showing the effects of 66 $\mathrm{Hz}$ stimulation of the anterior VTA on AMPAR EPSCs in all VTA DA neurons. Intra-VTA-evoked LTD was observed in 11 of 25 cells (44\%, Responders). $\boldsymbol{b}$, Time course of LTD in a representative VTA DA neuron. Inset, Mean EPSCs before and after $66 \mathrm{~Hz}$ stimulation of the VTA (scale bars, $10 \mathrm{~ms}, 200 \mathrm{pA}$ ). c, Absence of $66 \mathrm{~Hz}$ LTD (stimulation applied to the respective pathways at arrows) in the PPN-activated pathway, in the same VTA DA neurons demonstrating intra-VTA-initiated LTD. The $66 \mathrm{~Hz}$ stimulation was applied through the PPN electrode only when LTD was observed first in the intra-VTA pathway. LTD was never observed in the PPN pathway following $66 \mathrm{~Hz}$ stimulation of the VTA, nor following multiple bouts of $66 \mathrm{~Hz}$ stimulation to the PPN (arrows; $\boldsymbol{d}$ ). $\boldsymbol{d}$, Representative neuron demonstrating no effect of repeated $66 \mathrm{~Hz}$ stimulation of the PPN on either PPN- or intra-VTA-evoked EPSCs. $\boldsymbol{e}$, Mean time course of the effects of briefDHPG application (horizontal bar) on intra-VTA and PPN-evoked EPSCs in the same VTA DA neurons. JTx (500 nM), applied after DHPG washout, had no effect on either PPN or intra-VTA-evoked EPSCs, confirming the presence of GluR2-containing AMPARs at intra-VTA-activated synapses after LTD (compare with Fig. 1b). $\boldsymbol{f}$, Representative example of a cell in which DHPG caused robust LTD of the intra-VTA pathway, but had no effect on the PPN stimulated pathway, and the absence of a post-LTD JTx effect.

through PPN or intra-VTA stimulation in the same VTA DA neurons before and after JTx application. For intra-VTA-evoked EPSCs, the baseline RI was significantly larger than that observed after JTx application (control RI $=3.89 \pm 0.52, n=6$; JTx RI $=$ $2.08 \pm 0.28, n=6$; $p<0.01$, paired $t$ test), whereas the RIs for EPSCs evoked by PPN stimulation were unaffected by JTx (control RI $=3.1 \pm 0.56, n=3$; JTx RI $=2.67 \pm 1.1, n=3 ; p=0.55$, paired $t$ test; Fig. $1 c, d)$. No change was seen in RI before or after JTx application in the remaining intra-VTA- and PPN-evoked EPSCs $(n=5$, ns; Fig. $1 f)$. These data confirm that all PPN glutamate synapses contain GluR2 subunits, whereas many AMPAR synapses activated by intra-VTA stimulation lack GluR2, indicating that the subunit composition of AMPARs differs in a pathway-specific manner in VTA DA neurons. This would likely result in differences in synaptic strength between these pathways under baseline conditions.

\section{Pathway-specific LTD in VTA \\ DA neurons}

The distinct subunit composition of AMPARs targeted by PPN and intra-VTA afferents might be expected to respond differently to stimuli known to induce long-term changes in synaptic strength. Therefore, we examined the capacity of these pathways for LTD.

LTD of intra-VTA-activated EPSCs can be initiated in DA neurons by promoting glutamate overflow onto nonsynaptic type 1 metabotropic glutamate receptors (mGluR1) using short bursts of $66 \mathrm{~Hz}$ stimulation, or by application of the mGluR1 agonist DHPG (Bellone and Lüscher, 2005). We used both DHPG and $66 \mathrm{~Hz}$ stimulation to evaluate LTD in the PPN- and intra-VTA-activated pathways. Following a single $66 \mathrm{~Hz}$ stimulus train within the anterior VTA, 11 of 25 (44\%) VTA DA neurons demonstrated LTD of EPSCs evoked via this same pathway (Fig. $2 a, b)$, whereas the remaining 14 neurons did not show long-term changes (Fig. 2a).

Since the probability of observing intra-VTA-evoked $66 \mathrm{~Hz}$ LTD under these baseline conditions was relatively low, we attempted to induce LTD via the PPN pathway in only those neurons showing robust LTD following intraVTA stimulation. In these experiments, 10 min of baseline was recorded by alternating single stimuli between the intraVTA and PPN electrodes in the same neurons. A single $66 \mathrm{~Hz}$ stimulus train was then applied through the intra-VTA stimulator, followed by single pulse alternating stimulation of the pathways. If LTD of the intra-VTA-evoked EPSC was observed $10 \mathrm{~min}$ following the $66 \mathrm{~Hz}$ train, then $66 \mathrm{~Hz}$ stimulation was applied to the PPN, followed again by alternating single pulse stimulation. In these neurons $(n=$ 5), intra-VTA $66 \mathrm{~Hz}$ stimulation caused robust LTD (Fig. 2c), and this intra-VTA stimulation did not affect EPSCs evoked by single-pulse stimulation of the PPN (Fig. 2c), further demonstrating the independence of these two pathways (Good and Lupica, 2009). However, in contrast to the robust LTD observed in the intra-VTAactivated pathway, $66 \mathrm{~Hz}$ stimulation of the PPN did not result in LTD, or otherwise affect PPN-evoked EPSCs in any of these same neurons (Fig. 2c). Furthermore, in these same cells, multiple bouts of $66 \mathrm{~Hz}$ stimulation delivered to the PPN failed to initiate LTD of the PPN-evoked EPSCs in those cells demonstrating robust LTD of the VTA pathway (Fig. 2c).

These data suggest that, in contrast to AMPAR synapses activated by intra-VTA stimulation, the EPSCs evoked via PPN stimulation were incapable of undergoing LTD in the same neurons. However, the design of these experiments necessitated a $20 \mathrm{~min}$ 
delay in the application of $66 \mathrm{~Hz}$ stimulation to the PPN to permit assessment of LTD in the VTA pathway. Since this delay may have been sufficient to washout intracellular molecules necessary to initiate LTD, additional recordings were made in another group of VTA neurons $(n=9)$ in which $66 \mathrm{~Hz}$ stimulation was applied only through the PPN stimulator $\sim 10$ min after attaining whole-cell access (i.e., the same time as that used to initiate LTD following intra-VTA stimulation). LTD was not observed in any of these neurons following $66 \mathrm{~Hz}$ stimulation of the PPN (Fig. $2 d)$. Therefore, under control conditions, LTD was observed only after $66 \mathrm{~Hz}$ stimulation of the intra-VTA pathway, and not following the activation of glutamate afferents originating in the PPN.

The mGluR1 agonist DHPG can also induce LTD of EPSCs in VTA DA neurons activated via intra-VTA stimulation (Bellone and Lüscher, 2005). Similar to our result with $66 \mathrm{~Hz}$ stimulation described above, EPSCs activated via intraVTA stimulation demonstrated LTD in 20/25 neurons (80\%) following $5 \mathrm{~min}$ exposure of the slices to DHPG $(50 \mu \mathrm{M})$, whereas none $(0 / 11,0 \%)$ of the PPNevoked EPSCs were significantly altered by this drug (Fig. 2e,f).

Since mGluR-induced LTD is thought to occur when higher conductance, calciumpermeable, GluR2-lacking AMPARs are removed from activated synapses and replaced with lower conductance GluR2containing AMPA receptors (Mameli et al., 2007), we sought to determine the presence or absence of GluR2 at these AMPAR synapses using JTx after DHPGLTD was established in the intra-VTAactivated pathway. Despite the inhibition of intra-VTA-evoked EPSCs by JTx in the majority of neurons under baseline conditions (Fig. $1 b$ ), this polyamine did not affect EPSCs evoked via this pathway following DHPG-induced LTD $(n=4$; Fig. $2 e, f)$. Furthermore, as expected from experiments shown in Figure 1, JTx caused no change in the PPN-evoked EPSCs following DHPG exposure. This suggests that mGluR-LTD in the intra-VTA pathway was associated with an increase in synaptic GluR2-containing AMPARs.

These data demonstrate that under basal conditions AMPARs targeted by PPN glutamate afferents contain the GluR2 subunit, and do not exhibit $66 \mathrm{~Hz}$ or DHPG-induced LTD, whereas some AMPARs activated by intra-VTA stimulation appear to lack GluR2 and exhibit LTD. Thus the subcortical glutamatergic inputs to VTA DA neurons exhibit distinct functional properties compared with those activated via intra-VTA stimulation in the same cells. a
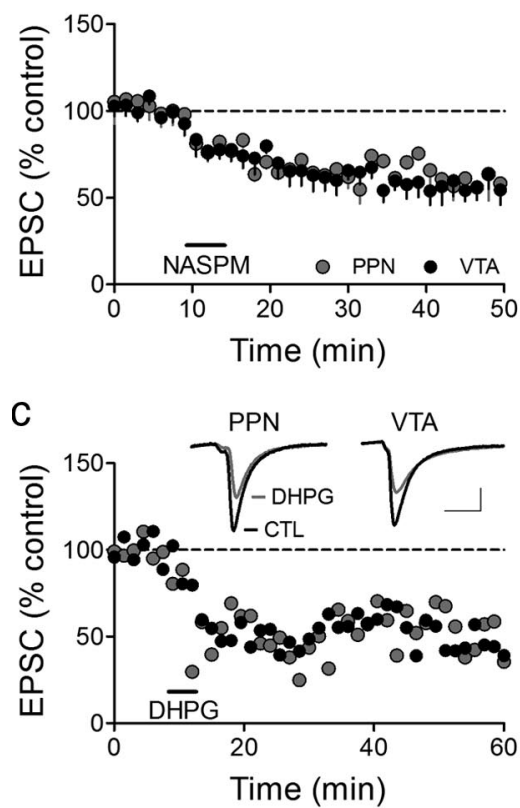

e
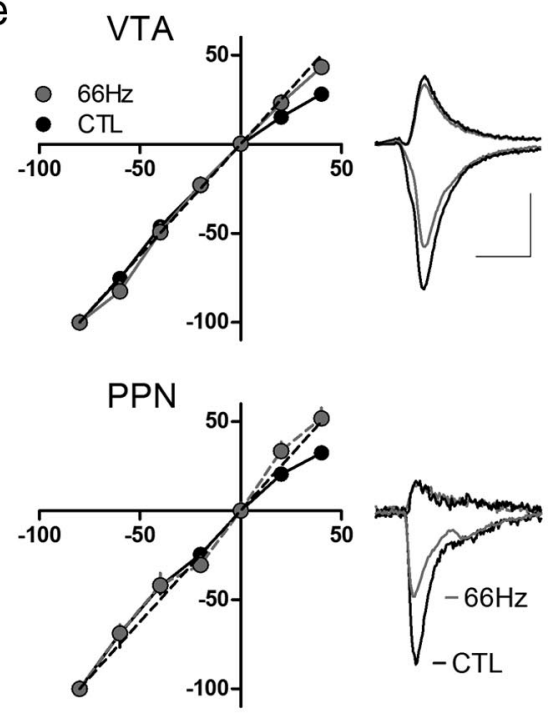

b

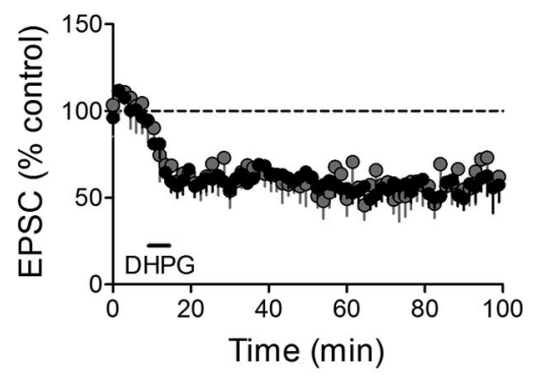

d

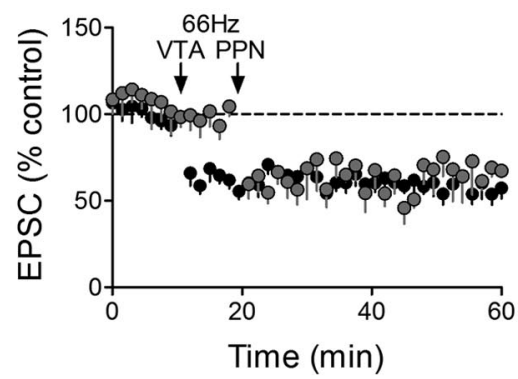

f

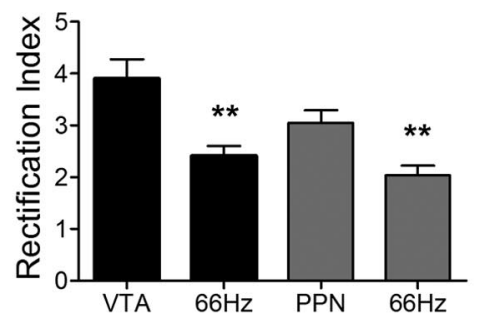

g

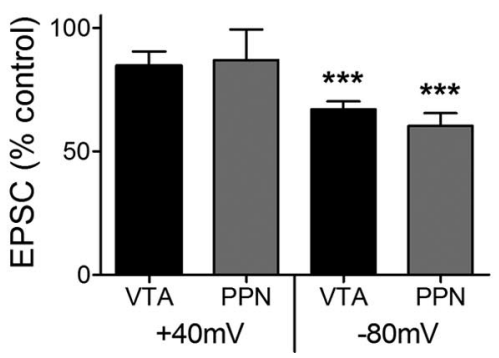

Figure 3. A single cocaine injection changes AMPAR subunit composition, leading to reliable LTD at both intra-VTA- and PPN-activated synapses in the same VTA DA neurons. $\boldsymbol{a}$, Mean time course of the effect of the JTx analog NASPM (horizontal bar) on EPSCs evoked via both pathways. PPN and the intra-VTA-activated glutamate synapses were inhibited by NASPM in all neurons, indicating a loss of GluR2-AMPARs following cocaine exposure. $\boldsymbol{b}$, Mean time course of DHPG-induced LTD of EPSCs evoked via both pathways $(n=6)$. LTD was observed at intra-VTA- and PPN-activated synapses in $100 \%$ of the cells $24 \mathrm{~h}$ after the cocaine injection. c, Representative time course and EPSCs from a single DA neuron showing DHPG-LTD in both pathways (scale bars, $5 \mathrm{~ms}, 100 \mathrm{pA}$ for VTA-evoked, and $5 \mathrm{~ms}, 25 \mathrm{pA}$ for PPN-evoked EPS(s). $\boldsymbol{d}$, Mean time course showing the effect of $66 \mathrm{~Hz}$ stimulation applied to the intra-VTA pathway, followed by PPN stimulation $10 \mathrm{~min}$ later $(n=8) . e$, Inward rectification of EPSCs in both pathways $24 \mathrm{~h}$ following a single cocaine exposure (CTL), and subsequent loss of rectification following $66 \mathrm{~Hz}$ stimulation in both pathways. EPSCS were normalized to those recorded at $-80 \mathrm{mV}$. Example wave forms are shown at $+40 \mathrm{mV}$ and $-80 \mathrm{mV}$ before and after $66 \mathrm{~Hz}$ stimulation in each pathway (scale bars, $5 \mathrm{~ms}, 200$ pA, VTA pathway; 5 ms, 50 pA, PPN pathway). $f$, Mean RI before and after $66 \mathrm{~Hz}$ stimulation of the intra-VTA- or PPN-activated inputs to the same VTA DA neurons. $\boldsymbol{g}$, Mean EPSC amplitude changes in both pathways at $+40 \mathrm{mV}$ and $-80 \mathrm{mV}$ after $66 \mathrm{~Hz}$ stimulation, normalized to pre- $66 \mathrm{~Hz}$ EPSC amplitudes ( $-80 \mathrm{mV}$, ANOVA, $\left.{ }^{* *} p<0.01,{ }^{* * *} p<0.0001\right)$.

\section{A single exposure to cocaine changes AMPAR subunit composition}

Exposure to cocaine in vivo can initiate the exchange of GluR2containing AMPARs for higher conducting GluR2-lacking AMPARs at intra-VTA-activated synapses (Bellone and Lüscher, 2006; Mameli et al., 2007), and this results in potentiation of these 


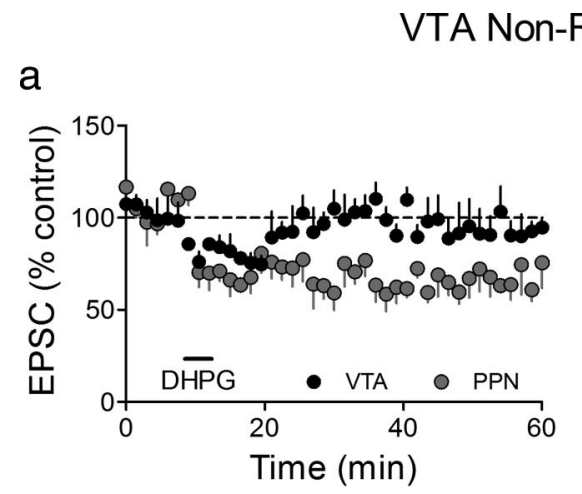

Responders

b

VTA Responders

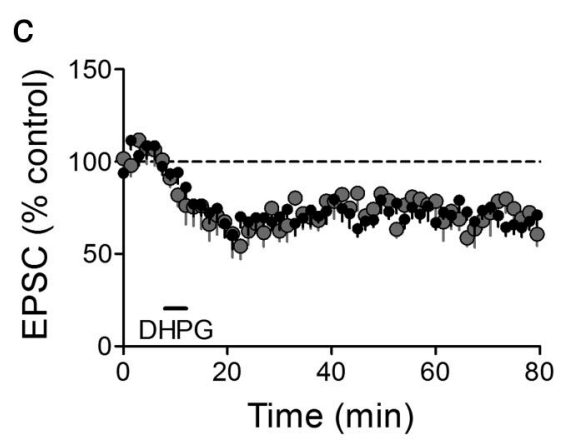

d
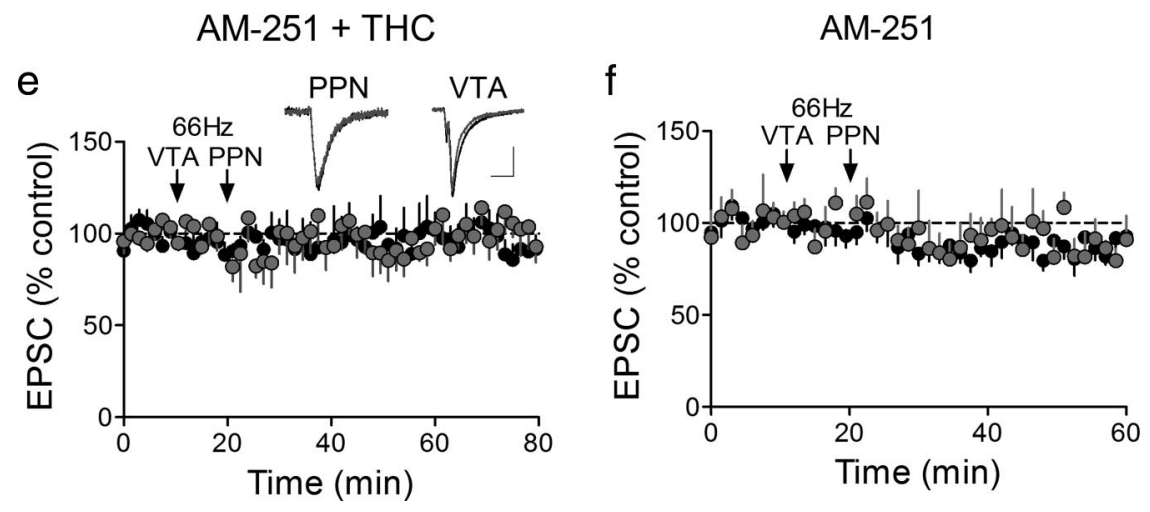

Figure 4. Afferent-specific LTD at PPN-glutamate synapses following a single exposure to $\Delta^{9}$-THC. $\boldsymbol{a}$, Mean time course showing that DHPG initiates LTD of PPN-evoked EPSCS $(n=16 / 16)$, but not of intra-VTA-evoked EPSCs, in the same subpopulation of VTA DA neurons $24 \mathrm{~h}$ after a single injection of $\Delta^{9}$-THC $(10 \mathrm{mg} / \mathrm{kg}$, i.p.). $\boldsymbol{b}$, Mean time course showing $66 \mathrm{~Hz}$ LTD of PPN-evoked EPSCS, but not intra-VTA-evoked EPSCs in a subpopulation of VTA DA neurons. c, DHPG-induced LTD of both pathways following a single injection of $\Delta^{9}$-THC in a subpopulation of VTA DA neurons. $\boldsymbol{d}$, Mean time course of $66 \mathrm{~Hz}$ LTD in both pathways in a subpopulation of neurons. $\boldsymbol{e}$, The CB1R antagonist AM251, injected 30 min before $\Delta^{9}$-THC blocks $66 \mathrm{~Hz}$ LTD. $f$, Mean time course of the effect of $66 \mathrm{~Hz}$ stimulation of both pathways in VTA DA neurons from animals receiving a single injection of AM251. In this experiment 6/14 (43\%) intra-VTA-evoked EPSCs demonstrated $66 \mathrm{~Hz}$ LTD, whereas 0/9 cells demonstrated LTD following $66 \mathrm{~Hz}$ stimulation of the PPN pathway.

synapses (Argilli et al., 2008). This effect of cocaine can then be reversed through reinsertion of the lower conducting GluR2containing AMPA receptors at these synapses following activation of mGluR1s, via either $66 \mathrm{~Hz}$ stimulation or application of DHPG (Bellone and Lüscher, 2006; Mameli et al., 2007). Since we found that JTx had no effect on the PPN glutamate synapse onto VTA DA neurons (Fig. 1), and LTD was not observed in this pathway (Fig. 2), we hypothesized that AMPARs containing GluR2 mediated these EPSCs under basal conditions, and that cocaine exposure might cause a switch from GluR2-containing to GluR2-lacking AMPARs at these PPN-activated synapses.

To investigate this, we measured the effects of NASPM (a synthetic analog of JTx) on both PPN- and intra-VTA-evoked responses in VTA DA neurons obtained from rats receiving a single intraperitoneal injection of cocaine $(15 \mathrm{mg} / \mathrm{kg}) 24 \mathrm{~h}$ before brain slice preparation. In contrast to JTx or NASPM, which had no effect on PPN-evoked EPSCs in slices from drugnaive animals (Fig. 1), NASPM reduced EPSCs that were evoked via both intraVTA $(n=7)$ or PPN stimulation $(n=6)$ in $100 \%$ of the cells obtained from cocaine-injected animals (Fig. 3a). This suggests that a single exposure to cocaine initiated the insertion of GluR2-lacking AMPARs into both synaptic pathways.

To determine whether this presumed change in AMPAR subunit composition following cocaine administration might also permit the more robust expression of LTD in these pathways we applied $66 \mathrm{~Hz}$ stimulation within the VTA or the PPN, or examined the response of these pathways to DHPG after a single injection of cocaine. Twenty-four hours after the cocaine injection, DHPG-induced LTD was observed in both the intra-VTA- and PPN-activated glutamatergic pathways in all of the VTA DA neurons $(6 / 6$, Fig. 3b,c). We next examined the effect of $66 \mathrm{~Hz}$ stimulation on the intra-VTA pathway, followed $10 \mathrm{~min}$ later by $66 \mathrm{~Hz}$ stimulation applied to the PPN pathway (8 neurons; Fig. $3 d$ ). In contrast to our initial experiments where $66 \mathrm{~Hz}$ LTD was never seen in the PPN-activated pathway (Fig. $2 a, c, d)$, LTD was now observed in both pathways in brain slices from animals receiving single cocaine injections, and this was accompanied by significant changes in the RI (intra-VTA stimulation, control $\mathrm{RI}=3.90 \pm 0.37$, post-LTD RI $=2.41 \pm$ $0.19, n=8, p<0.005$, paired $t$ test; PPN pathway, control RI $=3.05 \pm 0.24$, postLTD RI $=2.04 \pm 0.19, n=8, p<0.01$, paired $t$ test; Fig. $3 f$ ). This suggests that GluR-2 lacking AMPARs were inserted at intra-VTA- and PPN-activated synapses following a single exposure to cocaine, and that these higher conducting, $\mathrm{Ca}^{2+}$ permeable receptors were subsequently removed from these synapses during LTD, thus leading to a significantly smaller RI.

Collectively, these data demonstrate that a single exposure to cocaine can alter the AMPAR subunit composition of both subcortical PPN-activated, and locally activated synapses onto VTA DA neurons, resulting in more efficacious excitatory input that is then subject to modification by LTD.

\section{$\Delta^{9}$-THC induces a pathway-specific change in AMPA subunit composition}

Although prior studies have shown that GluR2-lacking AMPARs are inserted into intra-VTA-activated synapses on DA neurons after exposure to cocaine (Bellone and Lüscher, 2006), no studies have determined whether exposure to the psychoactive 
constituent of marijuana, $\Delta^{9}$-THC, affects these synapses, nor have comparisons among glutamatergic pathways been made. Therefore, rats were given single injections of $\Delta^{9}$-THC $(10 \mathrm{mg} / \mathrm{kg}$, i.p.) $24 \mathrm{~h}$ before preparation of brain slices, and the properties of intra-VTAand PPN-activated glutamatergic synapses were assessed in the same VTA DA neurons.

Unlike the data obtained following a single injection of cocaine, in which PPNand intra-VTA-activated glutamatergic synapses were similarly affected (Fig. 3), $\Delta^{9}$-THC exposure selectively altered the subcortical PPN afferent pathway. Specifically, following $\Delta^{9}$-THC exposure, DHPG-LTD was observed in only a portion (7/12 cells, 58\%) of the intra-VTAactivated synapses (Fig. $4 a, c$ ), but was seen in the PPN-activated pathway in every VTA DA neuron studied (13/13, 100\%; Fig. $4 a, c)$. Since the proportion of intraVTA-activated synapses demonstrating DHPG-induced LTD was not significantly different from that observed in control rats that were not injected with $\Delta^{9}$-THC (no $\Delta^{9}$-THC $=20 / 25$ vs $\Delta^{9}$ THC injected $=7 / 12 ; Z$ score $=0.99, \mathrm{~ns}$, $p=0.16)$, we conclude that the drug selectively enabled LTD of PPN glutamate synapses, without altering the expression of LTD in glutamate synapses activated via intra-VTA stimulation.

To determine whether this pathway-specific expression of LTD was also present with high-frequency stimulation, we first applied the $66 \mathrm{~Hz}$ stimulus the intra-VTA pathway, followed 10 min later by the PPN pathway. Consistent with the results observed with DHPG, $66 \mathrm{~Hz}$ stimulation applied to the intra-VTA pathway initiated LTD in only a portion of the cells $(11 / 20$ cells, $55 \%$ ), whereas the EPSCs elicited via PPN stimulation demonstrated LTD in all neurons (16/16; Fig. 4b,d). Also similar to the DHPG application was the observation that the proportion of cells demonstrating $66 \mathrm{~Hz}$ LTD, evoked via intra-VTA stimulation after $\Delta^{9}$-THC exposure (11/20), did not significantly differ from that observed in rats that had not been given $\Delta^{9}$-THC (16/ $30 ; Z=-0.174, p=0.57)$, further suggesting that the PPN glutamatergic synapses on VTA DA neurons were selectively altered by $\Delta^{9}$-THC.

To determine whether the $\Delta^{9}$-THC-induced increase in LTD susceptibility of the PPN glutamate pathway was mediated by the incorporation of GluR2-lacking AMPARs into these synapses, we measured the RI. In support of this mechanism, the RI of PPNevoked EPSCs was significantly larger after $\Delta^{9}$-THC treatment than in control neurons (control RI $=3.1 \pm 0.56, n=3$, post$\Delta^{9}$-THC RI $=4.22 \pm 0.55 ; n=7, p<0.05, t$ test $)$, and this value was significantly reduced in neurons from $\Delta^{9}$-THC-treated rats following $66 \mathrm{~Hz}$ LTD (post-LTD RI $=2.62 \pm 0.40 ; p<0.01$, paired $t$ test, Fig. $5 c$ ). Additionally, as expected, the baseline RI $(n=10,3.97 \pm 0.18)$ in the neurons demonstrating intra-VTA pathway LTD (VTA Responders) was significantly larger ( $p<$ 0.0003 , paired $t$ test) than that measured after $66 \mathrm{~Hz}$ LTD induction $(n=9,2.86 \pm 0.16$; Fig. $5 c)$, whereas in the cells that did not demonstrate LTD (VTA nonresponders) this value was not significantly affected (baseline RI $=3.45 \pm 0.25$; post $-\Delta^{9}$-THC $\mathrm{RI}=3.41 \pm 0.38, n=7, p>0.05$, paired $t$ test; Fig. $5 a$ ).

To determine whether the change in AMPAR subunit composition caused by $\Delta^{9}$-THC was mediated by cannabinoid receptors, another group of rats was injected with the cannabinoid CB1 receptor (CB1R) antagonist AM251 (2 mg/kg, i.p.), $30 \mathrm{~min}$ before the single $\Delta^{9}$-THC injection. LTD was not observed in any of the VTA DA neurons from animals given both AM251 and $\Delta^{9}$ THC injections, following $66 \mathrm{~Hz}$ stimulation of the PPN pathway $(n=5$, Fig. $4 e)$, confirming that the AMPAR subunit change initiated by $\Delta^{9}$-THC occurred as a result of CB1R activation by this drug. Furthermore, $66 \mathrm{~Hz}$ LTD was observed in a proportion of the VTA DA neurons using intra-VTA stimulation $24 \mathrm{~h}$ after the AM251 and $\Delta^{9}$-THC injections $(3 / 9,33 \%)$, and this proportion was not significantly different from that observed from control animals $(16 / 30,53 \% ; Z=0.67, \mathrm{~ns}, p=0.25)$. Additionally, AM251 alone had no effect on AMPAR subunit composition. Thus, in neurons from rats given a single injection with this drug, LTD was observed in only a portion of the intra-VTA-activated synapses $(6 / 14,43 \%)$, which did not differ from that observed in neurons from rats not receiving AM251 (16/30, 53\%; $Z=0.32, p=$ 0.37 ), and was never observed in the PPN-activated glutamate synapses in the same cells (0/9 cells; Figs. 4f, 6).

These data demonstrate that CB1Rs were activated by $\Delta^{9}$ THC, and this selectively caused the insertion of higher conducting GluR2-lacking AMPARs into PPN glutamatergic synapses. This change in AMPAR subunit composition was then permissive to LTD expression only in the PPN pathway. 


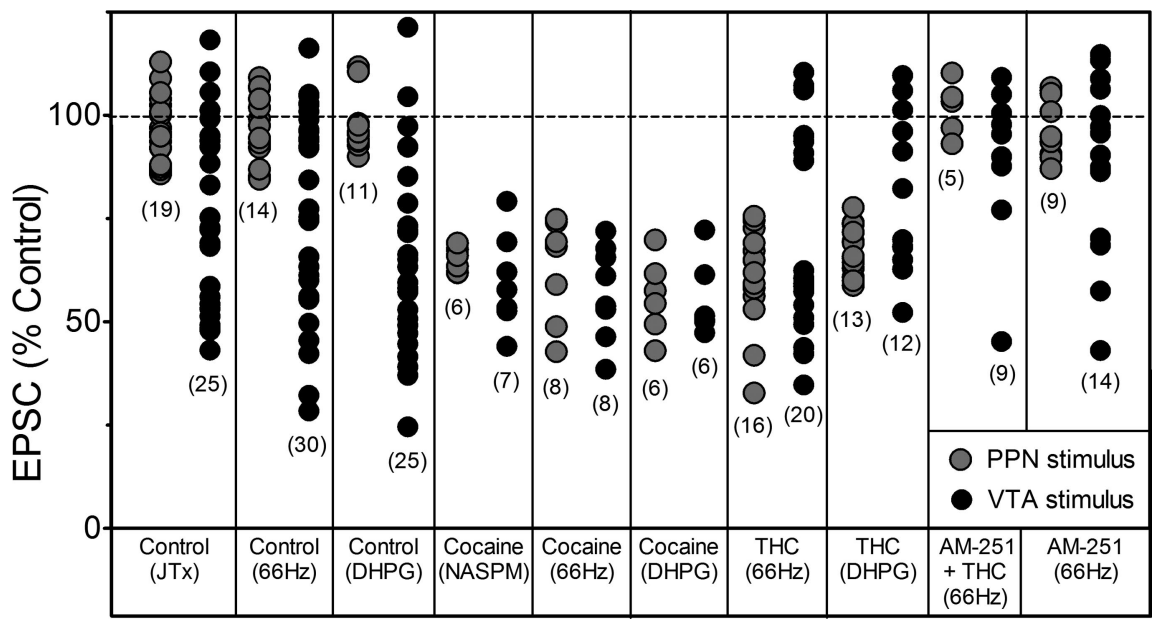

Figure 6. Compilation of the effects of all manipulations on EPSC amplitudes for all neurons included in this study. The numbers in parentheses represent the total number of VTA DA neurons in which observations were made during each manipulation. Gray circles indicate EPSCs evoked by stimulation of the PPN, whereas, black circles represent EPSCs evoked via stimulation of the rostral VTA (intraVTA). "Control" indicates either no injection $24 \mathrm{~h}$ before brain slice preparation, or those responses from animals receiving a single saline injection. Note that PPN-evoked EPSCs were insensitive to JTx and did not demonstrate $66 \mathrm{~Hz}$-, or DHPG-induced LTD unless measured in VTA DA neurons obtained from cocaine- or $\Delta^{9}$-THC-treated animals. In addition, $\Delta^{9}$-THC exposure did not appear to increase the likelihood of intra-VTA-evoked LTD expression above that observed under control conditions.

\section{Discussion}

The present study demonstrates that single VTA DA neurons are innervated by distinct glutamatergic afferents that activate either AMPARs containing the GluR2 subunit or AMPARs lacking this subunit. Thus, under baseline conditions, subcortical PPN glutamatergic afferents formed synapses on $\mathrm{Ca}^{2+}$-impermeable GluR2-containing AMPARs, whereas the afferents activated by conventional intra-VTA stimuli targeted $\mathrm{Ca}^{2+}$-permeable AMPARs that predominantly lacked this subunit. This demonstrates that pathway-specific differences in AMPAR subunit composition exist, and because prior results demonstrate that AMPARs lacking GluR2 subunits exhibit higher single channel conductances and greater permeability to $\mathrm{Ca}^{2+}$ (Swanson et al., 1997; Isaac et al., 2007), our data indicate that PPN inputs are likely weaker than those of other glutamate afferents to VTA DA neurons.

\section{Pathway-specific AMPAR subunit composition results in differential expression of LTD in VTA DA neurons}

Previous studies demonstrated that mGluR-dependent LTD at intraVTA-activated synapses on DA neurons is distinct from that observed in other brain regions, such as cerebellum and hippocampus (for review, see Lüscher and Huber, 2010). In these regions LTD results from the endocytosis of AMPARs (Argilli et al., 2008; Lüscher and Huber, 2010), whereas in VTA DA neurons LTD requires the exchange of GluR2-lacking AMPARs for those containing this subunit, following mGluR1 activation (Lüscher and Huber, 2010). Our study confirmed prior observations by showing that mGluR-LTD at the intra-VTA-evoked pathway was only observed when the AMPARs underlying these responses lacked the GluR2 subunit. Our data also extend these findings by demonstrating that the baseline capacity for LTD was input-specific, since glutamate synapses activated by subcortical PPN stimulation never exhibited LTD in the same neurons in which intra-VTA-activated LTD was observed. Based upon the prior observation that mGluR-dependent LTD in VTA DA neurons required synthesis and insertion of lowerconductance GluR2-containing AMPARs (Mameli et al., 2007), we hypothesize that our observed resistance of the PPN-activated path- way to LTD resulted from the presence of GluR2-containing AMPARs at these synapses under basal conditions. This was confirmed by the absence of polyamine sensitivity and lack of EPSC rectification. The presence of $\mathrm{Ca}^{2+}$-impermeable, lowerconductance, GluR2-containing AMPARs at PPN-activated synapses suggests that these afferents will have smaller effects on VTA DA neuron activity under basal conditions than other glutamate synapses lacking this subunit.

\section{A single exposure to cocaine decreases GluR2-containing AMPARs at PPN- and intra-VTA-activated glutamate} synapses on the same VTA DA neurons Under baseline conditions PPN-activated afferents targeted polyamine insensitive GluR2-containing AMPARs on VTA DA neurons. However, $24 \mathrm{~h}$ following a single cocaine injection, EPSCs evoked via this pathway were inhibited by NASPM, demonstrating a loss of GluR2-containing AMPARs. This was correlated with changes in rectification of PPN-activated EPSCs. Based on other studies using only intra-VTA stimulation, this switch from polyamine-insensitivity to full sensitivity in the PPN pathway likely resulted from a cocaine-induced exchange of GluR2-containing AMPARs for those lacking this subunit (Bellone and Lüscher, 2005; Mameli et al., 2007). The insertion of GluR2-lacking AMPARs at intra-VTA-activated synapses following cocaine treatment caused a marked increase in the incidence of both $66 \mathrm{~Hz}$ - and DHPG-induced LTD, such that all cells demonstrated this form of plasticity. However, the appearance of LTD at PPN-activated synapses was particularly notable because it was completely absent using $66 \mathrm{~Hz}$ or DHPG induction paradigms in control DA neurons, but was seen in all neurons $24 \mathrm{~h}$ after the single in vivo cocaine injection.

The observation that independent glutamatergic afferents underwent similar changes following cocaine exposure suggests that this drug broadly triggers the insertion of GluR2-lacking AMPARs at all glutamate synapses on VTA DA neurons, thereby priming these synapses for subsequent long-lived changes in synaptic strength upon activation by the appropriate afferent signal. This switch in AMPAR subunit composition resulting in increased EPSC amplitude has been likened to LTP, since cocaine exposure increases glutamatergic transmission, and occludes spike-timing-dependent LTP (Argilli et al., 2008). Consistent with this, we showed that cocaine potentiated glutamate synapses activated by intra-VTA and PPN stimulation. Since under basal conditions PPN inputs to VTA DA neurons targeted only lowconductance GluR2-containing AMPARs, the cocaine-induced potentiation of these PPN synapses would have a proportionally greater effect on DA neuron activity than intra-VTA-activated inputs. This is because there is a larger proportional change in the AMPARs underlying excitatory transmission in the PPN projection, and the strength of the PPN influence on VTA DA neurons is predicted to shift abruptly from weak to strong following cocaine exposure, whereas the intra-VTA-activated pathway would exhibit a smaller relative change. 


\section{Exposure to $\Delta^{9}$-THC selectively alters subcortical PPN glutamate inputs to VTA DA neurons}

Although the effects of several abused drugs on VTA DA neuron AMPARs have been examined (Faleiro et al., 2004; Bellone and Lüscher, 2006; Lane et al., 2008), the effects of $\Delta^{9}$-THC on AMPAR subunit composition has not been studied. As with cocaine, a single injection of $\Delta^{9}$-THC altered AMPAR subunit composition in VTA DA neurons. However, unlike the similar effects of cocaine on PPN- and intra-VTA-activated synapses, $\Delta^{9}$-THC caused a switch to GluR2-lacking AMPARs in only the PPN-activated pathway, and this was associated with the appearance of DHPG-LTD. Thus, although PPN-activated EPSCs never demonstrated LTD in control neurons, it was observed in all PPN-activated DA neurons following $\Delta^{9}$-THC. LTD was also specific to PPN-activated synapses, since the proportion of intraVTA-activated EPSCs demonstrating LTD did not change following $\Delta^{9}$-THC injection.

The permissive effect of $\Delta^{9}$-THC on PPN pathway LTD was mediated by CB1Rs since the antagonist AM251 prevented both LTD and changes in EPSC rectification. Although CB1Rs were implicated in the $\Delta^{9}$-THC effect, we did not determine whether these CB1Rs were located in the VTA, or in brain circuits that engage this nucleus. Previously, the potentiating effect of cocaine on intra-VTA-activated synapses was seen in as little as $3 \mathrm{~h}$ after cocaine exposure, either via a single injection, or through direct in vitro application (Argilli et al., 2008). Thus, cocaine could affect AMPARs through direct actions within the VTA, independently of interaction with circuits impinging upon the DA neurons (Argilli et al., 2008). This direct cocaine effect may explain the lack of selectivity with regard to PPN versus intra-VTA activated afferents in the present study, and further suggests that the more selective effect of $\Delta^{9}$-THC on the PPN projection may result either from altered output of this nucleus, or perhaps to a more direct action within the VTA that involves only PPN synapses. Previously, our laboratory and others have shown that CB1R agonists inhibit glutamatergic neurotransmission (Melis et al., 2004; Riegel and Lupica, 2004). However, the origin of the intra-VTA-activated glutamate afferents were not identified. In addition, $C B 1 R$ activation inhibits GABA inputs to $\mathrm{GABA}_{\mathrm{B}}$ (Riegel and Lupica, 2004), and $\mathrm{GABA}_{\mathrm{A}}$ receptors on VTA DA neurons (Szabo et al., 2002), and anatomical evidence has confirmed the presence of CB1Rs in the VTA (Mátyás et al., 2008). Therefore, although there is evidence that cocaine can alter AMPAR subunit composition via direct interaction in the VTA, it is unclear whether $\Delta^{9}$-THC effects on subcortically activated AMPARs occur locally, or via effects on VTA projecting nuclei. The present results suggest that a determination of CB1R actions on the PPN pathway is also warranted.

\section{Functional consequences of drug-induced AMPAR subunit changes}

An increase in the involvement of GluR2-lacking AMPARs at synapses on VTA DA neurons following exposure to abused drugs is expected to have important functional consequences for this central component of the brain reward circuitry. Thus, following single exposure to cocaine (Argilli et al., 2008) or $\Delta^{9}$-THC (present study), a switch to GluR2-lacking AMPARs will strengthen glutamatergic synaptic transmission. Furthermore, since GluR2-lacking receptors gate $\mathrm{Ca}^{2+}$, they may provide an additional mechanism through which intracellular $\mathrm{Ca}^{2+}$ levels can be increased to affect biochemical signaling processes (Carlezon and Nestler, 2002; Isaac et al., 2007). Our experiments, and others, demonstrate that brief exposure to abused drugs can alter AMPARs, which can then serve as a substrate for additional changes in synaptic efficacy through processes such as LTD. This suggests that altered AMPAR subunit composition represents a dynamic mechanism to mediate synaptic changes resulting from experience, including those occurring through passive exposure to, or self administration of abused drugs, and that this can lead to rapid adaptation of neuronal circuits (Carlezon and Nestler, 2002; Bellone and Lüscher, 2006; Kauer and Malenka, 2007; Mameli et al., 2007; Conrad et al., 2008). Since AMPAR subunit rearrangements in the VTA occur before those in other rewardrelevant brain nuclei, it is suggested that this may represent an early adaptation before recruitment of downstream targets, and the behavioral progression from casual drug use to addiction (Carlezon and Nestler, 2002; Mameli et al., 2007). We show that the involvement of GluR2-lacking AMPARs can be selectively increased at subcortical PPN glutamate synapses following exposure to $\Delta^{9}$-THC, and that this can provide a substrate to permit long-term changes in synaptic plasticity at these inputs. This, and our observation that cocaine can more broadly induce such changes at intra-VTA- and PPN-activated glutamate synapses, suggests that some abused drugs may recruit distinct glutamatergic circuits, and thereby selectively alter the degree of influence that certain brain nuclei have over VTA DA neuron function. Based on the pattern of AMPAR subunit changes following $\Delta^{9}$ THC exposure, we predict that unlike psychostimulants, subcortical glutamate inputs to VTA DA neurons will be more strongly and selectively affected by $\Delta^{9}$-THC, and we suggest that this may represent a neural code that permits the reward signal provided by $\Delta^{9}$-THC to be distinguished from other abused drugs.

\section{References}

Argilli E, Sibley DR, Malenka RC, England PM, Bonci A (2008) Mechanism and time course of cocaine-induced long-term potentiation in the ventral tegmental area. J Neurosci 28:9092-9100.

Bechara A, van der Kooy D (1989) The tegmental pedunculopontine nucleus: a brain-stem output of the limbic system critical for the conditioned place preferences produced by morphine and amphetamine. J Neurosci 9:3400-3409.

Bellone C, Lüscher C (2005) mGluRs induce a long-term depression in the ventral tegmental area that involves a switch of the subunit composition of AMPA receptors. Eur J Neurosci 21:1280-1288.

Bellone C, Lüscher C (2006) Cocaine triggered AMPA receptor redistribution is reversed in vivo by $\mathrm{mGluR}$-dependent long-term depression. Nat Neurosci 9:636-641.

Bonci A, Malenka RC (1999) Properties and plasticity of excitatory synapses on dopaminergic and GABAergic cells in the ventral tegmental area. J Neurosci 19:3723-3730.

Cameron DL, Wessendorf MW, Williams JT (1997) A subset of ventral tegmental area neurons is inhibited by dopamine, 5-hydroxytryptamine and opioids. Neuroscience 77:155-166.

Carlezon WA Jr, Nestler EJ (2002) Elevated levels of GluR1 in the midbrain: a trigger for sensitization to drugs of abuse? Trends Neurosci 25:610-615.

Conrad KL, Tseng KY, Uejima JL, Reimers JM, Heng LJ, Shaham Y, Marinelli M, Wolf ME (2008) Formation of accumbens GluR2-lacking AMPA receptors mediates incubation of cocaine craving. Nature 454:118-121.

Cornwall J, Cooper JD, Phillipson OT (1990) Afferent and efferent connections of the laterodorsal tegmental nucleus in the rat. Brain Res Bull 25:271-284.

Faleiro LJ, Jones S, Kauer JA (2004) Rapid synaptic plasticity of glutamatergic synapses on dopamine neurons in the ventral tegmental area in response to acute amphetamine injection. Neuropsychopharmacology 29:2115-2125.

Floresco SB, West AR, Ash B, Moore H, Grace AA (2003) Afferent modulation of dopamine neuron firing differentially regulates tonic and phasic dopamine transmission. Nat Neurosci 6:968-973.

Geisler S, Zahm DS (2005) Afferents of the ventral tegmental area in the rat-anatomical substratum for integrative functions. J Comp Neurol 490:270-294. 
Geisler S, Derst C, Veh RW, Zahm DS (2007) Glutamatergic afferents of the ventral tegmental area in the rat. J Neurosci 27:5730-5743.

Georges F, Aston-Jones G (2002) Activation of ventral tegmental area cells by the bed nucleus of the stria terminalis: a novel excitatory amino acid input to midbrain dopamine neurons. J Neurosci 22:5173-5187.

Gonon FG (1988) Nonlinear relationship between impulse flow and dopamine released by rat midbrain dopaminergic neurons as studied by in vivo electrochemistry. Neuroscience 24:19-28.

Good CH, Lupica CR (2009) Properties of distinct ventral tegmental area synapses activated via pedunculopontine or ventral tegmental area stimulation in vitro. J Physiol 587:1233-1247.

Grace AA, Onn SP (1989) Morphology and electrophysiological properties of immunocytochemically identified rat dopamine neurons recorded in vitro. J Neurosci 9:3463-3481.

Hyland BI, Reynolds JN, Hay J, Perk CG, Miller R (2002) Firing modes of midbrain dopamine cells in the freely moving rat. Neuroscience 114:475492.

Inglis WL, Olmstead MC, Robbins TW (2000) Pedunculopontine tegmental nucleus lesions impair stimulus-reward learning in autoshaping and conditioned reinforcement paradigms. Behav Neurosci 114:285-294.

Isaac JT, Ashby M, McBain CJ (2007) The role of the GluR2 subunit in AMPA receptor function and synaptic plasticity. Neuron 54:859-871.

Johnson SW, North RA (1992) Two types of neurone in the rat ventral tegmental area and their synaptic inputs. J Physiol 450:455-468.

Jones S, Kornblum JL, Kauer JA (2000) Amphetamine blocks long-term synaptic depression in the ventral tegmental area. J Neurosci 20:55755580.

Kauer JA, Malenka RC (2007) Synaptic plasticity and addiction. Nat Rev Neurosci 8:844-858.

Kitai ST, Shepard PD, Callaway JC, Scroggs R (1999) Afferent modulation of dopamine neuron firing patterns. Curr Opin Neurobiol 9:690-697.

Klitenick MA, Kalivas PW (1994) Behavioral and neurochemical studies of opioid effects in the pedunculopontine nucleus and mediodorsal thalamus. J Pharmacol Exp Ther 269:437-448.

Lane DA, Lessard AA, Chan J, Colago EE, Zhou Y, Schlussman SD, Kreek MJ, Pickel VM (2008) Region-specific changes in the subcellular distribution of AMPA receptor GluR1 subunit in the rat ventral tegmental area after acute or chronic morphine administration. J Neurosci 28:96709681.

Lüscher C, Huber KM (2010) Group 1 mGluR-dependent synaptic longterm depression: mechanisms and implications for circuitry and disease. Neuron 65:445-459.

Mameli M, Balland B, Luján R, Lüscher C (2007) Rapid synthesis and synaptic insertion of GluR2 for mGluR-LTD in the ventral tegmental area. Science 317:530-533.

Mansvelder HD, McGehee DS (2000) Long-term potentiation of excitatory inputs to brain reward areas by nicotine. Neuron 27:349-357.

Margolis EB, Lock H, Hjelmstad GO, Fields HL (2006) The ventral tegmental area revisited: Is there an electrophysiological marker for dopaminergic neurons? J Physiol 577:907-924.

Mátyás F, Urbán GM, Watanabe M, Mackie K, Zimmer A, Freund TF, Katona
I (2008) Identification of the sites of 2-arachidonoylglycerol synthesis and action imply retrograde endocannabinoid signaling at both GABAergic and glutamatergic synapses in the ventral tegmental area. Neuropharmacology 54:95-107.

Melis M, Pistis M, Perra S, Muntoni AL, Pillolla G, Gessa GL (2004) Endocannabinoids mediate presynaptic inhibition of glutamatergic transmission in rat ventral tegmental area dopamine neurons through activation of CB1 receptors. J Neurosci 24:53-62.

Nelson CL, Wetter JB, Milovanovic M, Wolf ME (2007) The laterodorsal tegmentum contributes to behavioral sensitization to amphetamine. Neuroscience 146:41-49.

Oakman SA, Faris PL, Kerr PE, Cozzari C, Hartman BK (1995) Distribution of pontomesencephalic cholinergic neurons projecting to substantia nigra differs significantly from those projecting to ventral tegmental area. J Neurosci 15:5859-5869.

Olmstead MC, Franklin KB (1993) Effects of pedunculopontine tegmental nucleus lesions on morphine-induced conditioned place preference and analgesia in the formalin test. Neuroscience 57:411-418.

Olmstead MC, Munn EM, Franklin KB, Wise RA (1998) Effects of pedunculopontine tegmental nucleus lesions on responding for intravenous heroin under different schedules of reinforcement. J Neurosci 18:50355044.

Omelchenko N, Sesack SR (2007) Glutamate synaptic inputs to ventral tegmental area neurons in the rat derive primarily from subcortical sources. Neuroscience 146:1259-1274.

Overton PG, Clark D (1997) Burst firing in midbrain dopaminergic neurons. Brain Res Brain Res Rev 25:312-334.

Riegel AC, Lupica CR (2004) Independent presynaptic and postsynaptic mechanisms regulate endocannabinoid signaling at multiple synapses in the ventral tegmental area. J Neurosci 24:11070-11078.

Saal D, Dong Y, Bonci A, Malenka RC (2003) Drugs of abuse and stress trigger a common synaptic adaptation in dopamine neurons. Neuron 37:577-582.

Schultz W (1998) Predictive reward signal of dopamine neurons. J Neurophysiol 80:1-27.

Swanson GT, Kamboj SK, Cull-Candy SG (1997) Single-channel properties of recombinant AMPA receptors depend on RNA editing, splice variation, and subunit composition. J Neurosci 17:58-69.

Szabo B, Siemes S, Wallmichrath I (2002) Inhibition of GABAergic neurotransmission in the ventral tegmental area by cannabinoids. Eur J Neurosci 15:2057-2061.

Ungless MA, Whistler JL, Malenka RC, Bonci A (2001) Single cocaine exposure in vivo induces long-term potentiation in dopamine neurons. Nature 411:583-587.

Wang HL, Morales M (2009) Pedunculopontine and laterodorsal tegmental nuclei contain distinct populations of cholinergic, glutamatergic and GABAergic neurons in the rat. Eur J Neurosci 29:340-358.

Wise RA (2005) Forebrain substrates of reward and motivation. J Comp Neurol 493:115-121.

Wolf ME, Sun X, Mangiavacchi S, Chao SZ (2004) Psychomotor stimulants and neuronal plasticity. Neuropharmacology 47 [Suppl 1]:S61-S79. 